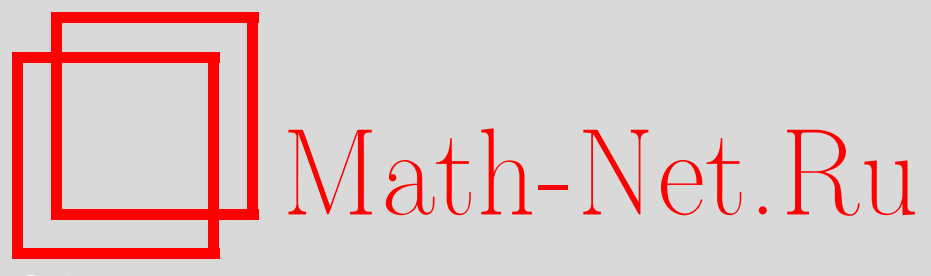

Н. А. В. Бедоя, Д. Л. Гонсалвес, Проблема разложимости разветвленных накрытий, Матем. сб., 2010, том 201, номер 12, 3-20

DOI: https://doi.org/10.4213/sm7572

Использование Общероссийского математического портала Math-Net.Ru подразумевает, что вы прочитали и согласны с пользовательским соглашением http://www . mathnet.ru/rus/agreement

Параметры загрузки:

IP: 35.173 .137 .237

26 апреля 2023 г., 18:11:16

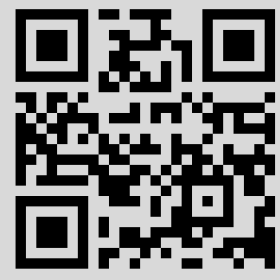




\author{
Н. А. В. Бедоя, Д. Л. Гонсалвес
}

\title{
Проблема разложимости разветвленных накрытий
}

\begin{abstract}
Как известно, заданное разветвленное накрытие степени $d$ между замкнутыми поверхностями определяет набор разбиений числа $d$, называемый данными ветвления. В работе показано, что любые данные ветвления реализуемы неразложимым примитивным разветвленным накрытием над связной замкнутой поверхностью $N$ такой, что ее эйлерова характеристика $\chi(N) \leqslant 0$. Отсюда следует, что разложимые и неразложимые реализации могут сосуществовать. Охарактеризованы также данные ветвления разложимых примитивных разветвленных накрытий.
\end{abstract}

Библиография: 20 названий.

Ключевые слова: разветвленные накрытия, группы перестановок.

\section{Введение}

Начнем с описания некоторых исторических фактов и результатов, связанных с проблемой, изучаемой в этой работе. В 1957 г. К. Борсук и Р. Молски поставили вопрос о существовании непрерывного отображения конечного порядка ${ }^{1}$, не являющегося композицией простых отображении, т.е. отображений порядка, не превосходящего двух (см. [1]). В 1959 г. К. Секлуцки показал, что каждое такое отображение, определенное на компактном конечномерном метрическом пространстве, является композицией простых отображений, и построил пример на бесконечномерном компактном пространстве, не являющийся композицией (см. [2]). В 1973 г. Дж. Бэйлдон показал, что если открытое сюръективное отображение конечного порядка между замкнутыми поверхностями является композицией $k$ простых открытых сюръективных отображений, то его порядок равен $2^{k}$ (см. [3]). В 2002 г. Е. Кжемпек в [4] построил для любого $n$ накрывающее отображение на локально линейно связном континууме, которое не разложимо в композицию накрывающих отображений порядка, не превосходящего $n-1$. В 2002 г. С. Богатая, С. Богатый и Х. Цишанг обобщили теорему Бэйлдона для композиций произвольных открытых отображений и показали, что порядок произведения (композиции) равен произведению порядков (см. [5]). Они также построили пример четырехлистного накрытия поверхности рода два поверхностью рода пять, которое не может быть разложено в композицию двух нетривиальных открытых отображений.

\footnotetext{
${ }^{1}$ Непрерывное отображение $\varphi$, определенное на пространстве $X$, называется отображением порядка, не превосходящего $k \in \mathbb{Z}^{+}$, если для любого $y \in \varphi(X)$ множество $\varphi^{-1}(y)$ содержит не более $k$ точек.
} 
В [6] Г. Уайбэрн показал, что открытые отображения конечного порядка на замкнутых поверхностях суть разветвленные накрытия. Цель настоящей работы - ответить на вопрос, является ли примитивное (сюръективное на $\pi_{1}$ ) разветвленное накрытие степени $d \in \mathbb{Z}^{+}$между замкнутыми связными поверхностями разложимым, т.е. являющимся композицией нетривиальных накрытий степени меньше $d$. Мы налагаем условие сюръективности на фундаментальной группе, так как непримитивное разветвленное накрытие всегда разложимо (см. [7]).

Разветвленное накрытие $\varphi: M \rightarrow N$ степени $d$ между замкнутыми связными поверхностями определяет конечный набор $\mathscr{D}$ разбиений числа $d$, т.е. данные ветвления. Обратно, если даны $\mathscr{D}$ и $N$, Д. Хьюзмоллер в [8] и К. Эзелл в [9] дали необходимое и достаточное условие (условие Гурвица для $\mathscr{D}$, см. п. 1.2) для существования разветвленного накрытия $\varphi: M \rightarrow N$ между связными замкнутыми поверхностями, имеющего данные ветвления $\mathscr{D}$, при условии $\chi(N) \leqslant 0$. В этом случае будем говорить, что $\mathscr{D}$ реализуем на $N$ отображением $\varphi$. Более того, С. Богатый, Д. Гонсалвес, Е. Кудрявцева и Х. Цишанг в [10] и [11] показали при том же условии, что разветвленное накрытие может быть выбрано примитивным.

Набор разбиений числа $d$, удовлетворяющий условию Гурвица, будем называть допустимым. Основным результатом настоящей работы является следующая теорема (теорема 3.3):

Теорема. Любые нетривиальные допустимые данные реализуемы неразложимым примитивным разветвленным накрытием над любой замкнутой связной поверхностъю $N$ такой, что $\chi(N) \leqslant 0$.

В работе также охарактеризованы допустимые данные, реализуемые разложимым примитивным разветвленным накрытием над $N$, т.е. разложимые данные на $N$, с помощью определения специального разложения данных, а именно, доказано следующее предложение (предложение 2.6).

ПреДЛОЖеНИЕ. При $\chi(N) \leqslant 0$ допустимые даннъе $\mathscr{D}$ разложимы на $N$ тогда и только тогда, когда имеется такое разложение данных $\mathscr{D}$, в котором первый множитель является нетривиальными допустимыми данными.

Задача, которую мы здесь решаем, является важной для понимания и возможной классификации разветвленных накрытий. После результатов работ [8] и [12] существенные результаты были получены в работах [7], [10], [11], [13], в которых проблема реализации была решена в классе примитивных (т.е. индуцирующих сюръекцию фундаментальных групп) накрытий. Мы продолжаем исследование проблемы реализации этого типа, изучая разложимость на (возможно, неразложимые) разветвленные накрытия. Рассматриваемая нами проблема также связана с обратной проблемой Галуа (см., например, [14] и [15]) и с построением примитивных и непримитивных групп монодромии, имеющимся в [16]. Кроме фактов, упомянутых выше, эта проблема кажется интересной и сама по себе. 
Статья состоит из введения и четырех параграфов. В 11 приведены основные определения и некоторые известные результаты, связанные с разветвленными накрытиями. В $\S 2$ дана характеризация данных ветвления разложимого примитивного разветвленного накрытия. В $\S 3$ сформулировано утверждение о том, что если $N$ является тором или бутылкой Клейна, то допустимое разбиение реализуемо на $N$ неразложимым примитивным разветвленным накрытием. Затем этот результат обобщен на любые допустимые данные и любую поверхность $N$ такую, что $\chi(N) \leqslant 0$. В $\S 4$ доказаны утверждения из $\S 3$.

Результаты, тесно связанные с этой работой, содержатся в диссертации $(\mathrm{PhD})$ первого автора, написанной под руководством профессора Д. Л. Гонсалвеса и выполненной при поддержке FAPESP (грант № 03/12309-4).

\section{§ 1. Предварительные результаты, терминология и обозначения}

1.1. О группах перестановок. Обозначим через $\Sigma_{d}$ симметрическую группу на множестве $\Omega$ из $d$ элементов, а через $1_{d}$ - ее единичный элемент. Если $\alpha \in \Sigma_{d}$ и $x \in \Omega$, то через $x^{\alpha}$ обозначим образ $x$ при действии $\alpha$. Конкретная перестановка $\alpha$ будет записываться или как произведение независимых циклов, или следующим образом:

$$
\alpha=\left(\begin{array}{lllc}
1 & 2 & \ldots & 2 k+1 \\
1^{\alpha} & 2^{\alpha} & \ldots & (2 k+1)^{\alpha}
\end{array}\right),
$$

в зависимости от того, как нам будет удобнее. Множество длин циклов в разложении на независимые циклы перестановки $\alpha$, включая тривиальные, определяет разбиение числа $d$, называемое ииклической структурой перестановки $\alpha$. Обозначим это разбиение через $D_{\alpha}=\left[d_{\alpha_{1}}, \ldots, d_{\alpha_{t}}\right]$ и определим $\nu(\alpha):=$ $\sum_{i=1}^{t}\left(d_{\alpha_{i}}-1\right)$. Тогда $\alpha$ называется четной перестановкой, если $\nu(\alpha) \equiv 0$ $(\bmod 2)$. Если дано разбиение $D$ числа $d$ и циклическая структура перестановки $\alpha$ есть $D$, то будем писать $\alpha \in D$ и полагать $\nu(D):=\nu(\alpha)$.

При $1<r \leqslant d$ перестановка $\alpha \in \Sigma_{d}$ называется $r$-ииклом, если в ее разложении на независимые циклы нетривиальный цикл единственный и имеет длину $r$. Перестановки $\alpha, \beta \in \Sigma_{d}$ называются сопряженными, если существует $\lambda \in \Sigma_{d}$ такая, что $\alpha^{\lambda}:=\lambda \alpha \lambda^{-1}=\beta$. Известно, что сопряженные перестановки имеют одну и ту же циклическую структуру.

Если $G$ - группа перестановок на множестве $\Omega$ и $x \in \Omega$, то определяются $n о д$ группа изотропии элемента $x, G_{x}:=\left\{g \in G: x^{g}=x\right\}$, и орбита элемента $x$ при действии $G, x^{G}:=\left\{x^{g}: g \in G\right\}$.

Для $H \subset G$ введем подмножества

$$
\begin{aligned}
\operatorname{Supp}(H) & :=\left\{x \in \Omega: x^{h} \neq x \text { для некоторого } h \in H\right\}, \\
\operatorname{Fix}(H) & :=\left\{x \in \Omega: x^{h}=x \text { для всех } h \in H\right\} .
\end{aligned}
$$

Для $\Lambda \subset \Omega$ и $g \in G$ положим $\Lambda^{g}:=\left\{y^{g}: y \in \Lambda\right\}$.

Говорят, что $G$ транзитивна, если для любой пары $x, y \in \Omega$ существует $g \in G$ такой, что $x^{g}=y$. Непустое подмножество $\Lambda \subset \Omega$ называется блоком 
транзитивной группы $G$, если для каждого $g \in G$ либо $\Lambda^{g}=\Lambda$, либо $\Lambda^{g} \cap \Lambda=\varnothing$. Блок $\Lambda$ называется тривиалъным, если либо $\Lambda=\Omega$, либо $\Lambda=\{x\}$ для некоторого $x \in \Omega$. Если $\Lambda$ - блок группы $G$, то множество $\Gamma:=\left\{\Lambda^{\alpha}: \alpha \in G\right\}$ определяет разбиение множества $\Omega$ на блоки. Это множество называется $c u$ стемой блоков, содержащей $\Lambda$, и количество элементов $\Lambda$ делит количество элементов $\Omega$. Группа $G$ естественно действует на $\Gamma$. Транзитивная группа перестановок называется примитивной, если она обладает только тривиальными блоками. В противном случае она называется непримитивной.

ПримеР 1.1. Транзитивная группа перестановок $G<\Sigma_{d}$, которая содержит $(d-1)$-цикл, примитивна. Действительно, без ограничения общности предположим, что $g=(1 \ldots d-1)(d) \in G$. Тогда любое собственное подмножество $\Lambda$ множества $\{1, \ldots, d\}$, содержащее $d$ и по меньшей мере еще один элемент, удовлетворяет условиям $\Lambda^{g} \neq \Lambda$ и $\Lambda^{g} \cap \Lambda \neq \varnothing$. Поэтому блоки группы $G$ тривиальны и $G$ примитивна.

ПримеР 1.2. Если НОД $(\ell, d)=1$ и $\ell$ превосходит любой нетривиальный делитель числа $d$ (это выполнено, например, при $d=2 \ell \pm 1$ ), то любая транзитивная группа перестановок $G<\Sigma_{d}$, содержащая $\ell$-цикл, примитивна. Действительно, пусть для определенности $G$ содержит цикл $(1, \ldots, \ell)$. Если $G$ имеет блок, содержащий два элемента $i$ и $j$ такие, что $i \leqslant \ell$ и $j>\ell$, то этот блок содержит также $1, \ldots, \ell$, откуда следует, что количество элементов блока больше или равно $\ell+1$ и, значит, оно равно $d$ и блок тривиален. В противном случае количество элементов любого блока делит $\ell$ и $d-\ell$, поэтому оно равно единице, откуда следует, что все блоки $G$ тривиальны. Следовательно, $G$ примитивная группа перестановок.

ПРЕДЛОжЕниЕ 1.3 (см. [17; следствие 1.5 A]). Пусть G- транзитивная группа перестановок на множестве $\Omega$, состоящем из не менее двух точек. Тогда $G$ примитивна тогда и только тогда, когда каждая подгруппа изотропии $G_{x}, x \in \Omega$, является максимальной подгруппой в $G$.

\section{2. О разветвленных накрытиях между замкнутыми поверхностя-}

ми. Сюръективное непрерывное открытое отображение $\varphi: M \rightarrow N$ между замкнутыми поверхностями такое, что:

1) при $x \in N$ множество $\varphi^{-1}(x)$ является дискретным;

2) существует непустое дискретное множество $B_{\varphi} \subset N$ такое, что ограничение $\widehat{\varphi}:=\left.\varphi\right|_{M-\varphi^{-1}\left(B_{\varphi}\right)}$ является обычным (неразветвленным) накрытием степени $d$,

называется разветвленным накрытием степени $d$ над $N$ и обозначается через $\left(M, \varphi, N, B_{\varphi}, d\right)$. Здесь $N$ - базовая поверхность, $M$ - накрывающая поверхность и $B_{\varphi}$ - множество точек ветвления. Его ассочиированное неразветвленное накрытие обозначается через $(\widehat{M}, \widehat{\varphi}, \widehat{N}, d)$, где $\widehat{N}:=N \backslash B_{\varphi}$ и $\widehat{M}:=M \backslash \varphi^{-1}\left(B_{\varphi}\right)$. Множество $B_{\varphi}-$ это просто образ тех точек из $M$, в которых $\varphi$ не является локальным гомеоморфизмом, поэтому каждая точка $x \in B_{\varphi}$ определяет нетривиальное разбиение $D_{x}$ числа $d$, состоящее из локальных степеней отображения $\varphi$ на каждой компоненте прообраза маленького круга $U_{x}$ 
вокруг $x$ такого, что $U_{x} \cap B_{\varphi}=\{x\}$. Набор $\mathscr{D}:=\left\{D_{x}\right\}_{x \in B_{\varphi}}$ называется данными ветвления, а его тотальный дефект - это натуральное число, определяемое формулой $\nu(\mathscr{D}):=\sum_{x \in B_{\varphi}} \nu\left(D_{x}\right)$. Тотальный дефект удовлетворяет формуле Римана-Гурвица (см. [12]):

$$
\nu(\mathscr{D})=d \chi(N)-\chi(M) .
$$

С разветвленным накрытием $\left(M, \varphi, N, B_{\varphi}, d\right)$ связывается группа перестановок, называемая группой монодромии отображения $\varphi,-$ это образ представления Гурвица

$$
\rho_{\varphi}: \pi_{1}\left(N \backslash B_{\varphi}, z\right) \rightarrow \Sigma_{d}
$$

которое переводит любой класс $\alpha \in \pi_{1}\left(N \backslash B_{\varphi}, z\right)$ в перестановку множества $\varphi^{-1}(z)=\left\{z_{1}, \ldots, z_{d}\right\}$, показывающую зависимость конечной точки поднятия петли из $\alpha$ от выбора начальной точки, где $z \in N \backslash B_{\varphi}$. В частности, для любой точки $x \in B_{\varphi}$ рассмотрим путь $c_{x}$ от точки $z$ до маленькой окружности $a_{x}$ вокруг $x$ и определим класс петель $\mathbf{u}_{x}:=\left[c_{x} a_{x} c_{x}^{-1}\right]$. Тогда циклическая структура перестановки $\alpha_{x}:=\rho_{\varphi}\left(\mathbf{u}_{x}\right)$ совпадает с $D_{x}$ и $\nu\left(\prod_{x \in B_{\varphi}} \alpha_{x}\right) \equiv \nu(\mathscr{D})$ $(\bmod 2)$.

Всюду далее $N$ будет обозначать связную замкнутую поверхность такую, что $\chi(N) \leqslant 0$. Тогда $N$ - это либо связная сумма $g \geqslant 1$ торов, $N=T_{g}$, либо связная сумма $g \geqslant 2$ проективных плоскостей, $N=P_{g}$. Если $B_{\varphi}=\left\{x_{1}, \ldots, x_{t}\right\}$, то мы используем следующие представления для соответствующих фундаментальных групп:

$$
\begin{aligned}
\pi_{1}( & \left.N \backslash B_{\varphi}, z\right) \\
r & = \begin{cases}\left\langle\mathbf{u}_{x_{1}}, \ldots, \mathbf{u}_{x_{t}}, a_{1}, b_{1}, \ldots, a_{g}, b_{g} \mid \mathbf{u}_{x_{1}} \cdots \mathbf{u}_{x_{t}}\left[a_{1}, b_{1}\right] \cdots\left[a_{g}, b_{g}\right]=1\right\rangle, & N=T_{g}, \\
\left\langle\mathbf{u}_{x_{1}}, \ldots, \mathbf{u}_{x_{t}}, a_{1}, \ldots, a_{g} \mid \mathbf{u}_{x_{1}} \cdots \mathbf{u}_{x_{t}} a_{1}^{2} \cdots a_{g}^{2}=1\right\rangle, & N=P_{g} .\end{cases}
\end{aligned}
$$

В частном случае, когда $N=P_{2}$ и $B_{\varphi}=\{x\}$, мы также работаем с представлением $\left\langle\mathbf{u}_{x}, a_{1}, a_{2} \mid \mathbf{u}_{x} a_{1} a_{2} a_{1} a_{2}^{-1}=1\right\rangle$, что будет ясно из контекста. Отметим, что перестановка $\rho_{\varphi}\left(\prod_{i=1}^{t} \mathbf{u}_{x_{i}}\right)$ всегда четная. Это необходимое условие известно как условие Гурвица и эквивалентно тому, что

$$
\nu(\mathscr{D}) \equiv 0(\bmod 2) .
$$

ТЕОРема 1.4 (см. [18]). Пусть даны $N$ и конечный набор $\mathscr{D}$ разбиений числа d. Предположим, что можно определить представление $\pi_{1}(N \backslash F, z) \rightarrow \Sigma_{d}$, аналогичное представлению $\rho_{\varphi}$, образ которого является транзитивной группой перестановок, где $F \subset N$ - конечное множество с тем же количеством элементов, что и $\mathscr{D}$. Тогда набор $\mathscr{D}$ реализуем на $N$.

TЕОРемА 1.5 (см. в [8] - ориентируемый случай; в [9] - неориентируемый случай). Если набор $\mathscr{D}$ допустим, то $\mathscr{D}$ реализуем на любой поверхности $N$ такой, что $\chi(N) \leqslant 0$.

ТЕОРема 1.6 (см. в [11] - геометрическая реализация; в [10] - алгебраическая реализация). Любые нетривиальные допустимые данные $\mathscr{D}$ реализуемы примитивным разветвленным накрытием над любой поверхностью $N$ такой, чmo $\chi(N) \leqslant 0$. 
Напомним, что накрытие называется разложимым, если его можно записать как композицию двух нетривиальных (т.е. имеющих степени больше единицы) накрытий; в противном случае накрытие называется неразложимым. В разложении разветвленного накрытия, не являющегося накрытием, по меньшей мере одна из компонент является разветвленным накрытием, не являющимся накрытием. Более того, поскольку степень разложимого накрытия равна произведению степеней его компонент (см. [5; теорема 2.3]), нас интересуют разветвленные накрытия составной степени.

Для упрощения обозначений в случае, когда фиксировано разветвленное накрытие $\left(M, \varphi, N, B_{\varphi}, d\right)$, отождествим: $\rho:=\rho_{\varphi}, G:=\operatorname{Im}(\rho)$. Напомним, что для фиксированной точки $z \in \widehat{N}$ группа $G$ - это группа перестановок множества $\varphi^{-1}(z)=\left\{z_{1}, \ldots, z_{d}\right\}$, а $G_{z_{i}}-$ подгруппа изотропии точки $z_{i}$.

Лемма 1.7. Пусть $\left(M, \varphi, N, B_{\varphi}, d\right)$-разветвленное накрытие. Тогда в последовательности

$$
\pi_{1}\left(\widehat{M}, z_{i}\right) \stackrel{\widehat{\varphi}_{\#}}{\longrightarrow} \pi_{1}(\widehat{N}, z) \stackrel{\rho}{\longrightarrow} G
$$

имеем $\rho^{-1}\left(G_{z_{i}}\right)=\widehat{\varphi}_{\#}\left(\pi_{1}\left(\widehat{M}, z_{i}\right)\right)$.

ДокАзАтельство. Рассмотрим петлю $\alpha$ класса $[\alpha] \in \rho^{-1}\left(G_{z_{i}}\right)$. Тогда $z_{i}^{\rho([\alpha])}=z_{i}$ и класс поднятия $\widetilde{\alpha}$ петли $\alpha$ с начальной точкой $z_{i}$ принадлежит $\pi_{1}\left(\widehat{M}, z_{i}\right)$ и $\widehat{\varphi}_{\#}([\widetilde{\alpha}])=[\alpha]$. Обратно, если $[\alpha] \in \widehat{\varphi}_{\#}\left(\pi_{1}\left(\widehat{M}, z_{i}\right)\right)$, то класс поднятия этой петли с начальной точкой $z_{i}$ принадлежит $\pi_{1}\left(\widehat{M}, z_{i}\right)$. Поэтому $\rho([\alpha]) \in G_{z_{i}}$. Лемма доказана.

Дадим теперь аналог теоремы Ритта (см. [19]) для разветвленных накрытий.

ПРеДЛОЖенИЕ 1.8. Разветвленное накрытие разложимо тогда и только тогда, когда его группа монодромии непримитивна.

ДокАзАтельство. Пусть $\left(M, \varphi, N, B_{\varphi}, d\right)$ разложимо. Тогда существуют поверхность $K$ и разветвленные накрытия $\psi, \eta$ степеней $w, u$ соответственно такие, что $\varphi=\eta \psi$ и $d=u w(u, w>1)$. Определим $\widehat{K}:=K-\eta^{-1}\left(B_{\varphi}\right)$. Пусть $\widehat{\varphi}, \widehat{\psi}, \widehat{\eta}$ - ограничения отображений $\varphi$ и $\psi$ на $\widehat{M}$ и отображения $\eta$ на $\widehat{K}$. Для фиксированной точки $z \in \widehat{N}$ рассмотрим точки $z_{1} \in \widehat{\varphi}^{-1}(z)$ и $y_{1}:=\widehat{\psi}\left(z_{1}\right)$. Имеем коммутативные диаграммы:
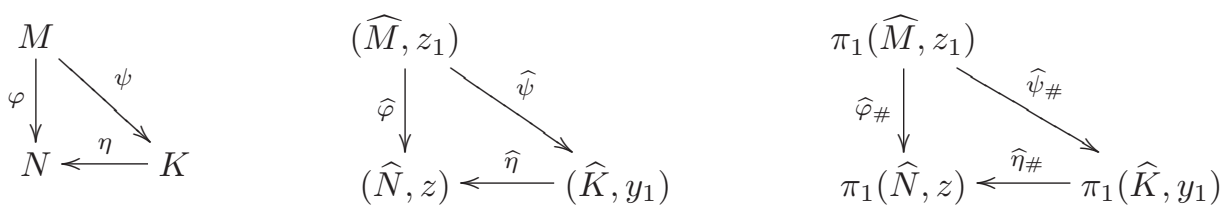

которые являются эквивалентами следующей последовательности собственных подгрупп:

$$
\widehat{\varphi}_{\#}\left(\pi_{1}\left(\widehat{M}, z_{1}\right)\right) \supsetneqq \widehat{\eta}_{\#}\left(\pi_{1}\left(\widehat{K}, y_{1}\right)\right) \supsetneqq \pi_{1}(\widehat{N}, z)
$$

с индексами

$$
\left|\widehat{\eta}_{\#}\left(\pi_{1}\left(\widehat{K}, y_{1}\right)\right): \widehat{\varphi}_{\#}\left(\pi_{1}\left(\widehat{M}, z_{1}\right)\right)\right|=w, \quad\left|\pi_{1}(\widehat{N}, z): \widehat{\eta}_{\#}\left(\pi_{1}\left(\widehat{K}, y_{1}\right)\right)\right|=u
$$


Применяя $\rho$ к (1.4) и используя лемму 1.7, мы получаем собственные подгруппы $G_{z_{1}} \supsetneqq \rho\left(\widehat{\eta}_{\#}\left(\pi_{1}\left(\widehat{K}, y_{1}\right)\right)\right) \supsetneqq G$ с такими же индексами, как и выше, поскольку $\operatorname{Ker}(\rho)<\rho^{-1}\left(G_{z_{1}}\right)=\widehat{\varphi}_{\#}\left(\pi_{1}\left(\widehat{M}, z_{1}\right)\right)$. Значит, подгруппа $G_{z_{1}}$ не является максимальной и по предложению 1.3 группа $G$ непримитивна.

Обратно, если $\rho: \pi_{1}(\widehat{N}, z) \rightarrow G$ - представление Гурвица для $\left(M, \varphi, N, B_{\varphi}, d\right)$ и $G$ непримитивна, то по предложению 1.3 существуют точка $z_{i} \in \varphi^{-1}(z)$ и собственная подгруппа $H_{1}<G$ такие, что

$$
\rho^{-1}\left(G_{z_{i}}\right) \supsetneqq \rho^{-1}\left(H_{1}\right) \supsetneqq \pi_{1}(\widehat{N}, z) .
$$

Пусть $u>1$ - индекс $\rho^{-1}\left(H_{1}\right)$ в $\pi_{1}(\widehat{N}, z)$ и $(\widehat{K}, \widehat{\eta}, \widehat{N}, u)$ - неразветвленное накрытие, отвечающее подгруппе $\rho^{-1}\left(H_{1}\right)$. Так как $\rho^{-1}\left(G_{z_{i}}\right)=\widehat{\varphi}_{\#}\left(\pi_{1}\left(\widehat{M}, z_{i}\right)\right)($ см. лемму 1.7$)$, то $\widehat{\varphi}$ имеет поднятие $\widehat{\psi}: \widehat{M} \rightarrow \widehat{K}$ и мы получаем коммутативную диаграмму неразветвленных накрытий. Мы хотим продолжить ее на $M, N$ и компактификацию $K$ для $\widehat{K}$. Это продолжение получается применением теории накрывающих пространств к ограничениям диаграммы на маленькие окружности вокруг элементов из $B_{\varphi}$. Предложение доказано.

Говорят, что отображение ориентирующе правильно, если оно переводит сохраняющие ориентацию петли в сохраняющие ориентацию, а меняющие ориентацию петли - в меняющие ориентацию петли. Разветвленное накрытие является ориентирующе правильным отображением (см. [13]).

ЗАмЕчАниЕ. При $g \geqslant 1$ подгруппа кручения абеленизованной группы $\pi_{1}\left(P_{g}\right)$ является циклической группой порядка два. В представлении

$$
\pi_{1}\left(P_{g}\right)=\left\langle a_{1}, \ldots, a_{g} \mid a_{1}^{2} \cdots a_{g}^{2}=1\right\rangle
$$

эта подгруппа группы $\pi_{1}\left(P_{g}\right)_{a b}$ порождена единственным элементом $a_{1}+\cdots+a_{g}$ порядка два.

ПреДЛОЖЕНИЕ 1.9. Пусть $\left(K, \eta, N, B_{\eta}, u\right)$ - примитивное разветвленное накрытие не являющееся гомеоморфизмом и $\chi(N) \leqslant)$. Тогда для любого $w \in \mathbb{N}$ существует подгруппа $H$ в $\pi_{1}(K)$ индекса $w$ такая, что $\left.\eta_{\#}\right|_{H}$ - эпиморфизм.

ДокАЗАТЕЛЬСтво. В силу теоремы об изоморфизме (см. [20; гл. 1, теорема 1.8]) и того, что $\eta_{\#}-$ эпиморфизм, достаточно работать с абеленизованными группами. Если $N=T_{g}$, то в силу формул $(1.1),(1.3)$ и неравенств $\nu(D)>0$ и $d \geqslant 2$ имеем $K=T_{h}$ для некоторого $h>g>0$. Пусть $\bar{\eta}_{\#}: \pi_{1}\left(T_{h}\right)_{a b} \rightarrow$ $\pi_{1}\left(T_{g}\right)_{a b}$ - эпиморфизм абеленизованных фундаментальных групп, индуцированный $\eta_{\#}$. Заметим, что $\pi_{1}\left(T_{h}\right)_{a b} \cong\left\langle a_{1}, \ldots, a_{2(h-g)}, b_{1}, \ldots, b_{2 g}\right\rangle$, где $a_{i}$ - образующие $\operatorname{Ker}\left(\bar{\eta}_{\#}\right)$ и $\bar{\eta}_{\#}\left(b_{j}\right)$ - образующие $\pi_{1}\left(T_{g}\right)_{a b}$. Определим подгруппу $\bar{H}=\left\langle w a_{1}, a_{2}, \ldots, a_{2(h-g)}, b_{1}, \ldots, b_{2 g}\right\rangle$ индекса $w$. Ограничение $\left.\bar{\eta}_{\#}\right|_{\bar{H}}$ является эпиморфизмом.

Если $N=P_{g}$ и $g \geqslant 2$, то поскольку $\eta$ примитивно и ориентирующе правильно, обязательно $K=P_{h}$ и $h \geqslant 2 g$ в силу (1.1) и (1.3). Так как $\pi_{1}(K)_{a b}$ и $\pi_{1}(N)_{a b}$ имеют изоморфные подгруппы кручения (обе $\mathbb{Z}_{2}$, см. замечание), то ограничение эпиморфизма $\bar{\eta}_{\#}$ на подгруппы кручения является изоморфизмом. Факторизуя $\pi_{1}(K)_{a b}$ и $\pi_{1}(N)_{a b}$ по их подгруппам кручения, получаем эпиморфизм 
между свободными абелевыми группами рангов $h-1$ и $g-1$, из чего следует, что $\operatorname{Ker}\left(\bar{\eta}_{\#}\right)$ - свободная абелева группа ранга $h-g$. Поэтому можно выбрать такой набор образующих $\left\{a_{1}, \ldots, a_{h}\right\}$ для $\pi_{1}\left(P_{h}\right)_{a b}$, что $\operatorname{Ker}\left(\bar{\eta}_{\#}\right)=\left\langle a_{1}, \ldots, a_{h-g}\right\rangle-$ свободная абелева группа ранга $h-g$. В частности, $a_{1}$ имеет бесконечный порядок. Ограничение эпиморфизма $\bar{\eta}_{\#}$ на подгруппу $\bar{H}=\left\langle w a_{1}, a_{2}, \ldots, a_{h}\right\rangle$ индекса $w$ является эпиморфизмом. Предложение доказано.

\section{§ 2. Характеризация разложимых данных}

В определении понятия, которое мы будем сейчас изучать, в допустимые данные будем включать тривиальные разбиения (состоящие из одних лишь единиц). Отметим, что они не меняют тотальный дефект. Пусть $u, w, s \in \mathbb{N}$, $U=\left[u_{1}, \ldots, u_{s}\right]-$ разбиение числа $u$ и $\mathscr{W}=\left\{W_{1}, \ldots, W_{s}\right\}-$ набор разбиений числа $w$. Определим произведение-разбиение $U . \mathscr{W}$ как разбиение числа $u w$, полученное умножением на $u_{i}$ каждой компоненты разбиения $W_{i}$ и взятием объединения по всем $i=1, \ldots, s$.

ПримеР 2.1. При $u=w=3$ разбиение $[2,2,2,1,1,1]$ представимо в виде произведения-разбиения числа 9 следующими способами: его можно представить либо как

$$
[1[2,1], 1[2,1], 1[2,1]]=U \cdot \mathscr{W}, \quad U=[1,1,1], \quad \mathscr{W}=\{[2,1],[2,1],[2,1]\},
$$

либо как

$$
[2[1,1,1], 1[1,1,1]]=U \cdot \mathscr{W}, \quad U=[2,1], \quad \mathscr{W}=\{[1,1,1],[1,1,1]\} .
$$

ОПРЕДЕЛЕНИЕ 2.2. Пусть $\mathscr{U}=\left\{U_{1}, \ldots, U_{t}\right\}$ - семейство разбиений числа $u$, где разбиение $U_{i}$ содержит $s_{i}$ элементов, $\mathscr{W}=\bigcup_{i=1}^{t} \mathscr{W}_{i}$ - объединение наборов разбиений числа $w$, где $\mathscr{W}_{i}$ - набор из $s_{i}$ разбиений. Тогда определим $\mathscr{U} . \mathscr{W}$ как набор из $t$ разбиений числа $u w$, где $i$-е разбиение является произведением $U_{i} . \mathscr{W}_{i}$, $1 \leqslant i \leqslant t$. Если дан набор $\mathscr{D}$ разбиений числа $d$ и нетривиальное разложение числа $d$ на множители (например, $d=u w$ ), и если существуют $t \in \mathbb{N}, \mathscr{U}=$ $\left\{U_{1}, \ldots, U_{t}\right\}$ и $\mathscr{W}=\bigcup_{i=1}^{t} \mathscr{W}_{i}$ такие, что

$$
\mathscr{D}=\mathscr{U} . \mathscr{W}=\left\{U_{i} \cdot \mathscr{W}_{i}\right\}_{i=1}^{t},
$$

то будем говорить, что $\mathscr{U}=\left\{U_{1}, \ldots, U_{t}\right\}$ и $\mathscr{W}=\bigcup_{i=1}^{t} \mathscr{W}_{i}$ определяют алгебраическое разложение (или разложение на множители) данных $\mathscr{D}$.

Пример 2.3. Пусть $d \in \mathbb{N}$ - нечетное составное число. Тогда любое нетривиальное разложение числа $d$ на два натуральных множителя, $d=u w$, определяет разложение допустимых данных $\mathscr{D}=\{[d]\}$ на допустимые нетривиальные множители $\mathscr{U}=\{[u]\}$ и $\mathscr{W}=\{[w]\}$.

ПреДЛОЖЕНИЕ 2.4. Пустъ $d=u w ~ u \mathscr{D}, \mathscr{U}, \mathscr{W}-$ наборы разбиений чисел $d$, $u, w$ соответственно такие, что $\mathscr{D}=\mathscr{U} . \mathscr{W}$. Тогда $\nu(\mathscr{D})=\nu(\mathscr{W})+w \nu(\mathscr{U})$. 
ДоказАтельство. Если $\mathscr{D}=\mathscr{U} . \mathscr{W}$, то существуют натуральные числа $t$ и $s_{i}, i=1, \ldots, t$, такие что $\mathscr{U}=\left\{U_{1}, \ldots, U_{t}\right\}$ и $\mathscr{W}=\bigcup_{i=1}^{t} \mathscr{W}_{i}$, где $U_{i}$ - разбиение числа $u$ на $s_{i}$ компонент и $\mathscr{W}_{i}-$ набор из $s_{i}$ разбиений числа $w, i=1, \ldots, t$. Тогда $\mathscr{D}=\left\{U_{i} \cdot \mathscr{W}_{i}\right\}_{i=1}^{t}$ и

$$
\begin{aligned}
\nu(\mathscr{D}) & =\sum_{i=1}^{t} \nu\left(U_{i} . \mathscr{W}_{i}\right)=\sum_{i=1}^{t}\left(u w+\nu\left(\mathscr{W}_{i}\right)-w s_{i}\right)=\sum_{i=1}^{t} \nu\left(\mathscr{W}_{i}\right)+\sum_{i=1}^{t} w\left(u-s_{i}\right) \\
& =\nu(\mathscr{W})+w \sum_{i=1}^{t} \nu\left(U_{i}\right)=\nu(\mathscr{W})+w \nu(\mathscr{U}) .
\end{aligned}
$$

Предложение доказано.

СлеДСтвиЕ 2.5. Множители в разложении допустимых (т.е. имеющих четный дефект) данных на множители не обязательно являются допустимыми .

Напомним, что набор разбиений числа $d$ называется разложимым на $N$, если он реализуем на $N$ разложимым примитивным $d$-листным разветвленным накрытием.

ПреДЛОжЕНИЕ 2.6. Пусть $N$ - связная замкнутая поверхность такая, что $\chi(N) \leqslant 0$. Допустимые данные $\mathscr{D}$ разложимы на $N$ тогда и только тогда, когда существует такое разложение данных $\mathscr{D}$, в котором первый множитель является нетривиальными допустимыми данными.

ДокАЗАТЕЛЬСтво. Предположим, что $\left(M, \varphi, N, B_{\varphi}, d\right)$ - разложимое примитивное разветвленное накрытие, реализующее $\mathscr{D}$. Тогда существуют поверхность $K$ и накрытия $\psi, \eta$ степеней $w, u$ соответственно такие, что $\varphi=\eta \psi$. Следовательно, $d=u w$ и, поскольку $\varphi$ примитивно, существует непустое подмножество $B_{\eta} \subset B_{\varphi}$ такое, что $\left(K, \eta, N, B_{\eta}, u\right)$ - примитивное разветвленное накрытие с данными ветвления $\widetilde{\mathscr{U}}$. Заметим, что каждая точка $x \in B_{\varphi}$ определяет разбиение числа $u$ (тривиальное в случае $\left.x \in B_{\varphi} \backslash B_{\eta}\right)$ и каждая точка из $\eta^{-1}(x)$ определяет разбиение числа $w$ (тривиальное, если такая точка не является точкой ветвления для $\psi$ ). Другими словами, $x \in B_{\varphi}$ определяет разбиение $U_{x}$ числа $u$ и набор $\mathscr{W}_{x}$ разбиений числа $w$ такие, что $U_{x} . \mathscr{W}_{x}$ является разбиением числа $d$, определяемым точкой $x$ для $\varphi$. Тогда $\mathscr{D}=\left\{U_{x} . \mathscr{W}_{x}\right\}_{x \in B_{\varphi}}$ является разложением с допустимым нетривиальным первым множителем $\mathscr{U}=\left\{U_{x}\right\}_{x \in B_{\varphi}}$, так как $\nu(\mathscr{U})=\nu(\widetilde{\mathscr{U}})$ (различия между $\mathscr{U}$ и $\widetilde{\mathscr{U}}$ состоят лишь в тривиальных разбиениях) и $B_{\eta} \neq \varnothing$.

Предположим теперь, что $d=u w$, и пусть $\mathscr{D}=\left\{U_{x} . \mathscr{W}_{x}\right\}_{x \in B}$ - такое разложение допустимых данных, в котором первый множитель $\mathscr{U}$ нетривиален и допустим, где $B \subset N$ - конечное подмножество. По теореме 1.6 существует примитивное разветвленное накрытие $\left(K, \eta, N, B_{\eta}, u\right)$, реализующее $\mathscr{U}$. В частности, $B_{\eta} \subset B$ и $U_{x}$ - тривиальное разбиение для каждой точки $x \in B \backslash B_{\eta}$. По предложению 2.4 второй множитель $\mathscr{W}$ (возможно, тривиальный) допустим. Если он нетривиален, то по теореме 1.6 существует примитивное разветвленное накрытие $\left(M, \psi, K, B_{\psi}, w\right)$, реализующее его как данные ветвления. Без 
ограничения общности мы можем предполагать, что $B_{\psi} \subset \eta^{-1}(B)$ и что для каждого $x \in \eta\left(B_{\psi}\right)$ выполнено $U_{x}=\left[u_{x, 1}, \ldots, u_{x, s_{x}}\right], \eta^{-1}(x)=\left\{y_{x, 1}, \ldots, y_{x, s_{x}}\right\}$, $\eta$ имеет локальную степень $u_{x, j}$ в $y_{x, j}$, где $\psi$ и точка $y_{x, j}$ определяют разбиение $\mathscr{W}_{x, j}$ числа $w, 1 \leqslant j \leqslant s_{x}$. Итак, $B_{\psi} \subset \eta^{-1}\left(A \cup B_{\eta}\right)$. Таким образом, $\left(M, \eta \psi, N, B_{\eta} \cup \eta\left(B_{\psi}\right), d=u w\right)$ - разложимое примитивное разветвленное накрытие с данными ветвления $\mathscr{D}$. Если $\mathscr{W}$ тривиален, то требуемый результат следует из предложения 1.9. Предложение 2.6 доказано.

СледСтвие 2.7. Если данные $\mathscr{D}=\left\{D_{1}, \ldots, D_{t}\right\}$ разложимы на $N$, то для любого $i=1, \ldots, t$ каждая компонента разбиения $D_{i}$ является произведением двух натуральных чисел (при этом одно из них или они оба могут быть равны единице), не превосходящих степеней двух накрытий из разложения соответственно.

ПримеР 2.8. Примитивное разветвленное накрытие вида $\left(M, \varphi, T_{1},\{x\}, 4\right)$ неразложимо: заметим, что возможные допустимые данные суть $[1,3]$ и $[2,2]$. Но $[1,3]$ не реализуемо разложимым примитивным разветвленным накрытием на $T_{1}$ по следствию 2.7 , а каждое разложение данных $[2,2]$ имеет либо тривиальный, либо недопустимый первый множитель на $T_{1}$.

\section{§ 3. Реализация данных ветвления неразложимыми разветвленными накрытиями}

Начнем этот параграф с примера допустимых данных, которые допускают разложимую и неразложимую реализации на $T_{1}$ одновременно.

ПримеР 3.1. Рассмотрим реализуемые данные

$$
\mathscr{D}=\{[3,2,2,2],[3,2,2,2]\}=\{[1[3], 2[1,1,1]],[1[3], 2[1,1,1]]\}
$$

на $T_{1}$ с нетривиальным допустимым первым множителем $\mathscr{U}=\{[1,2],[1,2]\}$ и вторым множителем $\mathscr{W}=\bigcup_{i=1}^{2}\{[3],[1,1,1]\}_{i}$. Согласно предложению 2.6 данные $\mathscr{D}$ разложимы на $T_{1}$, но, с другой стороны, мы можем определить следующее представление:

$$
\begin{aligned}
& \rho: \pi_{1}\left(T_{1} \backslash\{x, y\}\right)=\left\langle a, b, \mathbf{u}_{\mathbf{x}}, \mathbf{u}_{\mathbf{y}} \mid[a, b] \mathbf{u}_{\mathbf{x}} \mathbf{u}_{\mathbf{y}}=1\right\rangle \rightarrow \Sigma_{9}, \\
& a \longmapsto(145678932) \text {, } \\
& b \longmapsto(24567893) \text {, } \\
& \mathbf{u}_{\mathbf{x}} \longmapsto\left(\begin{array}{lll}
1 & 23
\end{array}\right)(45)(67)(89) \text {, } \\
& \mathbf{u}_{\mathbf{y}} \longmapsto\left(\begin{array}{lll}
1 & 2 & 3
\end{array}\right)(45)(67)(89) \text {, }
\end{aligned}
$$

где $G=\operatorname{Im}(\rho)$ - транзитивная примитивная группа перестановок, так как она содержит 9- и 8-циклы (см. пример 1.1). Тогда согласно предложению 1.8 определяемое ею разветвленное накрытие неразложимо (и, следовательно, примитивно).

Цель этого параграфа - показать, что любые допустимые данные реализуемы неразложимым (и, следовательно, примитивным) разветвленным накрытием на связной замкнутой поверхности $N$ такой, что $\chi(N) \leqslant 0$. В теореме 3.2 
(которая будет доказана в следующем параграфе) это утверждается для случая, когда $N$ является либо тором $T_{1}$, либо бутылкой Клейна $P_{2}$ с ровно одной точкой ветвления. Эта теорема будет использована для доказательства в общем случае.

Tеорема 3.2. Пусть $d \in \mathbb{N}$ - составное число и $D=\left[d_{1}, \ldots, d_{t}\right]-$ нетривиальное разбиение числа $d$ такое, что $\nu(D) \equiv 0(\bmod 2)$. Тогда существуют неразложимые (, , следовательно, примитивные) разветвленные накрытия над тором $T_{1}$ и над бутылкой Клейна $P_{2}$ соответственно, реализующие $D$ как данные ветвления.

Теорема 3.3. Любые нетривиальные допустимые данные реализуемы неразложимым (и, следовательно, примитивным) разветвленным накрытием над любой поверхностъю $N$ такой, что $\chi(N) \leqslant 0$.

ДокАЗАтеЛьство. Пусть $\mathscr{D}=\left\{D_{1}, \ldots, D_{r}\right\}-$ допустимые данные. При каждом $i=1, \ldots, r$ выберем перестановку $\gamma_{i} \in \Sigma_{d}$, циклическая структура которой равна $D_{i}$.

Если $\prod_{i=1}^{t} \gamma_{i} \neq 1_{d}$, то циклическая структура этого произведения является нетривиальным разбиением $D=\left[d_{1}, \ldots, d_{t}\right]$ числа $d$ таким, что $t<d$ и $\nu(D) \equiv \nu(\mathscr{D}) \equiv 0(\bmod 2)$. Таким образом, по теореме 3.2 оно реализуемо неразложимым (и, следовательно, примитивным) разветвленным накрытием над $T_{1}$ (над $P_{2}$ соответственно). По предложению 1.8 существуют перестановки $\lambda, \beta \in \Sigma_{d}(\omega, \theta$ соответственно) такие, что циклическая структура перестановки $[\lambda, \beta]\left(\omega \theta \omega \theta^{-1}\right.$ или $\omega^{2} \theta^{2}$ соответственно) есть $D$ и группа перестановок $G_{1}:=\langle\lambda, \beta\rangle\left(G_{2}:=\langle\omega, \theta\rangle\right.$ соответственно) транзитивна и примитивна. Итак, для $N=S_{g}$ определим представление:

$$
\rho_{1}:\left\langle\left\{a_{i}, b_{i}\right\}_{i=1}^{g} \cup\left\{\mathbf{u}_{\mathbf{j}}\right\}_{j=1}^{t} \mid \prod_{j=1}^{t} \mathbf{u}_{\mathbf{j}}=\prod_{j=0}^{g-1}\left[b_{g-j}, a_{g-j}\right]\right\rangle \rightarrow \Sigma_{d},
$$

переводящее $a_{1} \longmapsto \beta, b_{1} \longmapsto \lambda, \mathbf{u}_{\mathbf{i}} \longmapsto \gamma_{i}$ и $\left\{a_{j}, b_{j}\right\}_{j=2}^{g} \longmapsto 1_{d}$ (соответственно для $N=P_{g}, g>1$, определим представление

$$
\rho_{2}:\left\langle\left\{a_{i}\right\}_{i=1}^{g} \cup\left\{\mathbf{u}_{\mathbf{j}}\right\}_{j=1}^{t} \mid \prod_{j=1}^{t} \mathbf{u}_{\mathbf{j}}=\prod_{j=0}^{g-1} a_{g-j}^{2}\right\rangle \rightarrow \Sigma_{d},
$$

переводящее $a_{1} \longmapsto \theta, a_{2} \longmapsto \omega, \mathbf{u}_{\mathbf{i}} \longmapsto \gamma_{i}$ и $\left.\left\{a_{j}\right\}_{j=3}^{g} \longmapsto 1_{d}\right)$. Так как $G_{1}<$ $\operatorname{Im}\left(\rho_{1}\right)$, то группа $\operatorname{Im}\left(\rho_{1}\right)$ транзитивна и примитивна. Таким образом, по предложению 1.8 определяемое ею примитивное разветвленное накрытие неразложимо (аналогично для $G_{2}$ ).

Если $\prod_{i=1}^{t} \gamma_{i}=1_{d}$ и существует некоторая перестановка $\gamma_{i}$ с циклом длины больше или равной трем, мы заменим $\gamma_{i}$ на $\gamma_{i}^{-1}$. Если $d>2$ и каждая $\gamma_{i}$ есть произведение независимых циклов длин меньше или равных двум, мы заменим один символ в одном цикле длины два символом из другого цикла. Итак, мы не меняем циклическую структуру перестановок $\gamma_{i}$, а новое произведение $\prod_{i=1}^{t} \gamma_{i}$ отлично от $1_{d}$, и мы приходим к предыдущему случаю. Если $d=2$, то 
данные $\mathscr{D}$, очевидно, реализуемы на любой поверхности $N$; соответствующее разветвленное накрытие неразложимо, поскольку число два - простое. Теорема доказана.

\section{§4. Доказательство теоремы 3.2}

Пусть $d \in \mathbb{N}$ - составное число и $D=\left[d_{1}, \ldots, d_{t}\right]$ - нетривиальное разбиение числа $d$ такое, что $\nu(D)=d-t \equiv 0(\bmod 2)$. Согласно предложению 1.8 достаточно доказать существование перестановок $\lambda, \beta, \omega, \theta \in \Sigma_{d}$ таких, что перестановки $[\lambda, \beta]$ для $T_{1}$ и либо $\omega \theta \omega \theta^{-1}$, либо $\omega^{2} \theta^{2}$ для $P_{2}$ имеют циклическую структуру $D$, а группы перестановок $G_{1}:=\langle\lambda, \beta\rangle$ и $G_{2}:=\langle\omega, \theta\rangle$ транзитивны и примитивны. Проведем доказательство отдельно в каждом из следующих трех случаев:

1) $t=1$;

2) $D=[2, \ldots, 2]$;

3) $t>1$ и $d_{i} \neq 2$ для некоторого $i \in\{1, \ldots, t\}$.

Перестановки, определенные в [10; доказательства теоремы 2.2 и теоремы 2.3 , случай $r=1$ ], работают в случае 1) (см. ниже). Для случаев 2) и 3) мы будем использовать следующую идею: для заданной перестановки $\alpha \in D$ определим перестановку $\beta \in \Sigma_{d}$ такую, что $\beta$ и $\alpha \beta$ имеют одну и ту же циклическую структуру, а группа перестановок $H:=\langle\alpha, \beta\rangle<\Sigma_{d}$ транзитивна и примитивна. Тогда существует $\lambda \in \Sigma_{d}$ такая, что $\alpha=[\lambda, \beta]$ и группа $\langle\lambda, \beta\rangle$ тоже транзитивна и примитивна (так как содержит $H$ ). Аналогично, так как $\alpha \beta$ и $\beta^{-1}$ сопряжены, то существует $\omega \in \Sigma_{d}$ такая, что $\alpha \beta=\omega \beta^{-1} \omega^{-1}$. Определим $\theta=\omega^{-1} \beta^{-1}$. Тогда $\alpha=\omega^{2} \theta^{2}$ и группа $\langle\omega, \theta\rangle$ транзитивна и примитивна.

Случай 1). Если $t=1$, то $D=[d]$ и $d=2 k+1$ для некоторого $k>0$. Для $T_{1}$ определим перестановки $\lambda=(k+1 k+2 \ldots 2 k 2 k+1)$ и

$$
\beta=\left(\begin{array}{ccccccc}
1 & \ldots & k & k+1 & k+2 & \ldots & 2 k+1 \\
2 k+1 & \ldots & k+2 & k+1 & 1 & \ldots & k
\end{array}\right) .
$$

Тогда $[\lambda, \beta]=(12 \ldots k k+1 k+2 \ldots 2 k+1)$ имеет циклическую структуру $D$ и группа $G_{1}=\langle\lambda, \beta\rangle$ транзитивна. Согласно примеру 1.2 при $d=2 k+1$ и $\ell=k+1$ группа перестановок $G_{1}$ примитивна. Для $P_{2}$ определим $\omega=\lambda$,

$$
\theta=\left(\begin{array}{ccccccc}
1 & 2 & \ldots & k+1 & k+2 & \ldots & 2 k+1 \\
2 k+1 & k+1 & \ldots & 2 k & 1 & \ldots & k
\end{array}\right)
$$

Тогда $\omega \theta \omega \theta^{-1}=\left(\begin{array}{l}1 \\ 1\end{array} 2 k+1\right)$ имеет циклическую структуру $D$ и аналогично ориентируемому случаю мы заключаем, что группа перестановок $G_{2}=\langle\omega, \theta\rangle$ транзитивна и примитивна.

Случай 2). Если $D=[2, \ldots, 2]$, то $d=2 t$ для некоторого четного $t$. Пусть

$$
\begin{aligned}
& \alpha=\left(\begin{array}{ll}
1 & 2
\end{array}\right)(34) \cdots(t-1 t) \cdots(2 t-12 t) \in D, \\
& \beta=\left(\begin{array}{lllll}
1 & 3 & \ldots & t-1
\end{array} \ldots 2 t-14\right)(26 \ldots t \ldots 2 t) \text {, }
\end{aligned}
$$


т.е. первый цикл в $\beta$ состоит из возрастающей последовательности нечетных чисел от 1 до $2 t-1$ и приписанного к ней четного числа 4 , а второй цикл состоит из возрастающей последовательности всех четных чисел от 2 до $2 t$, кроме числа 4. Индукцией по $t$ можно показать, что $\beta$ и $\alpha \beta$ сопряжены, поэтому существует $\lambda \in \Sigma_{d}$ такая, что $\alpha \beta=\lambda \beta \lambda^{-1}$. Итак, $[\lambda, \beta]=\alpha$ имеет циклическую структуру $D$. Рассмотрим транзитивную группу $G_{1}:=\langle\lambda, \beta\rangle$. Пусть $\|1\|$ означает нетривиальный блок группы $G_{1}$, содержащий элемент 1 . Если $\|1\| \subset 1^{\langle\beta\rangle}$ содержится в орбите элемента 1 при действии $\langle\beta\rangle$, то его число элементов \# $\|1\|$ является общим делителем чисел $t+1$ и $2 t$, откуда следует, что $\#\|1\|=1$. С другой стороны, если $\|1\|$ содержит элементы обоих циклов перестановки $\beta$, то НОД $(t+1, t-1) \neq 1$, что невозможно. Так как блоки тривиальны, то $G_{1}$ примитивна и случай поверхности $T_{1}$ разобран. Для поверхности $P_{2}$ мы используем идею, описанную в наброске доказательства в начале этого параграфа, чтобы определить $\omega, \theta \in \Sigma_{d}$ такие, что $\alpha=\omega^{2} \theta^{2}$. Так как $G_{2}:=\langle\omega, \theta\rangle=\langle\omega, \beta\rangle$ транзитивна и $\beta$ обеспечивает примитивность группы $G_{1}$, то $G_{2}$ тоже примитивна.

Случай 3). Наконец, предположим, что $t>1$ и $d_{i} \neq 2$ для некоторого $i \in\{1, \ldots, t\}$. Определим

$$
\delta_{0}:=0, \quad \delta_{i}:=\sum_{j=1}^{i} d_{j}, \quad C_{i}:=\left(\delta_{i-1}+1 \ldots \delta_{i}\right) \in \Sigma_{d}
$$

и последовательность $\Delta_{i}:=\left\{\delta_{i-1}+k\right\}_{k=2}^{d_{i}}$ при $i=1, \ldots, t$. Так как $t<d$, мы можем взять $d_{1}>1$, откуда следует, что $\Delta_{1} \neq \varnothing$. Без ограничения общности положим

$\alpha:=\prod_{i=1}^{t} C_{i}=(1 \underbrace{2 \ldots \delta_{1}}_{\Delta_{1}})(\delta_{1}+1 \underbrace{\delta_{1}+2 \ldots \delta_{2}}_{\Delta_{2}}) \cdots(\delta_{t-1}+1 \underbrace{\delta_{t-1}+2 \ldots \delta_{t}}_{\Delta_{t}}) \in D$.

Так как имеется $d_{i} \neq 2$, то либо $\operatorname{Fix}(\alpha) \neq \varnothing$, либо $\alpha^{2} \neq 1_{d}$. Определим $d$-цикл

$$
\beta:=\left(1 \delta_{1}+1 \delta_{2}+1 \ldots \delta_{t-1}+1 \Delta_{1} \Delta_{2} \ldots \Delta_{t}\right) .
$$

Обозначим через $E_{i}, O_{i}$ возрастающие последовательности четных и нечетных элементов в $\overline{\operatorname{Supp}\left(C_{i}\right)}:=\left\{\delta_{i-1}+1\right\} \cup \Delta_{i}$ соответственно. Заметим, что $\alpha \beta$ является $d$-циклом, полученным последовательным присоединением этих последовательностей друг за другом, а именно,

$$
\alpha \beta= \begin{cases}\left(O_{1} E_{2} O_{3} \ldots E_{t} E_{1} O_{2} E_{3} \ldots O_{t}\right), & \text { если } t \text { четно, } \\ \left(O_{1} E_{2} O_{3} \ldots O_{t} E_{1} O_{2} E_{3} \ldots E_{t}\right), & \text { если } t \text { нечетно. }\end{cases}
$$

Здесь мы используем то, что $t$-я последовательность равна либо $E_{t} \ni d$ при четном $t$, либо $O_{t} \ni d$ при нечетном $t$, а потому она непуста и заканчивается элементом $d$. Следовательно, за ней стоит $E_{1}$. Значит, $\alpha \beta$ и $\beta$ сопряжены и существует $\lambda \in \Sigma_{d}$ такая, что $\alpha \beta=\lambda \beta \lambda^{-1}$ и $\alpha=[\lambda, \beta]$. 
Пусть $H:=\langle\alpha, \beta\rangle$. Она, очевидно, транзитивна. Мы утверждаем, что группа перестановок $H$ примитивна (по меньшей мере, после подходящей перестановки чисел $d_{1}, \ldots, d_{t}$, см. ниже). Предположим противное, т.е. пусть существует нетривиальный делитель $n$ числа $d$ такой, что $H$ имеет систему из $n$ блоков с $d / n$ элементами в блоке. Тогда справедливы следующие утверждения I)-VIII).

I) $\|1\|=\left\|1^{\beta^{n}}\right\|$ и $\|i\|=\left\{i, i^{\beta^{n}}, i^{\beta^{2 n}}, \ldots, i^{\beta^{(d / n-1) n}}\right\}=\left\{i^{\beta^{k n}} \mid k \in \mathbb{Z}\right\}$ для любого $i \in\{1, \ldots, d\}$. Поэтому $R:=\left\{1,1^{\beta}, 1^{\beta^{2}}, \ldots, 1^{\beta^{n-1}}\right\}$ является множеством представителей всех блоков и последовательные элементы в $\beta$ находятся в разных блоках.

ДокАЗАТЕЛЬСтво. Поскольку $\beta$ является $d$-циклом, блоки полностью определяются циклами перестановки $\beta^{n}$.

II) $n<t$ (по меньшей мере, в случае $d_{1}=\max \left\{d_{1}, \ldots, d_{t}\right\}, d_{2}=\min \left\{d_{1}, \ldots, d_{t}\right\}$ и $\left.d_{3}=\max \left\{d_{3}, \ldots, d_{t}\right\}\right)$. Поэтому $R=\left\{1, \delta_{1}+1, \delta_{2}+1, \ldots, \delta_{n-1}+1\right\}, 1^{\beta^{n}}=\delta_{n}+1$ и $\Gamma:=\left\{\|1\|,\left\|\delta_{1}+1\right\|, \ldots,\left\|\delta_{n-1}+1\right\|\right\}$ является системой всех блоков.

ДокАзАтЕльство. Если $n \geqslant t$, то существует $i \in\{1, \ldots, t\}$ такое, что $1^{\beta^{n}} \in \Delta_{i}$

$$
\beta=(1 \underbrace{\delta_{1}+1 \ldots \delta_{i}+1 \ldots \delta_{t-1}+1 \Delta_{1} \Delta_{2} \ldots \Delta_{i} \ldots \Delta_{t}}_{n}) .
$$

Если $i>1$, то $1^{\alpha^{-1}}$ и $1^{\beta^{n} \alpha^{-1}}$ оба из $R$, находятся в одном и том же блоке в силу первого утверждения в I), что противоречит второму утверждению в I). Если $i=1$ (и поэтому $1^{\beta^{n}}=n-t+2$ ) и существует $d_{j}>2$, то обязательно

$$
d_{j} \leqslant n-t+2=1^{\beta^{n}} \in \Delta_{1}=\left\{2, \ldots, d_{1}\right\}
$$

поскольку $d_{1}=\max \left\{d_{1}, \ldots, d_{t}\right\}$. Действительно, в противном случае положим $j=1$ и, применяя перестановку $\alpha$ к элементам 1 и $1^{\beta^{n}}$, получим элементы $1^{\alpha}=2=1^{\beta^{t}}$ и $1^{\beta^{n} \alpha}=n-t+3=1^{\beta^{n+1}}$ в $\Delta_{1}$, в одном и том же блоке, что в силу I) дает $n \mid(t-1)$, поэтому $n<t$ или $t=1$. Получаем противоречие. Поэтому $1^{\beta^{n}}=n-t+2=d_{1}$, откуда следует, что $1^{\alpha}=2=1^{\beta^{t}}<d_{1}=1^{\beta^{n}}$ и $1^{\beta^{n}} \alpha=d_{1}^{\alpha}=1$, они оба из $R$ и являются различными элементами из одного блока. Получаем противоречие с I). Если $i=1$ и $d_{j} \leqslant 2$ при $j=1, \ldots, t$, то $\Delta_{j}$ содержит не более одного элемента и $\alpha^{2}=1$. Поэтому $\operatorname{Fix}(\alpha) \neq \varnothing$ и $\alpha$ является произведением четного числа транспозиций, поскольку $\nu(D) \equiv 0(\bmod 2)$. Отсюда $C_{1}=(12)$,

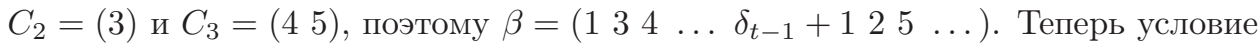
$i=1$ дает соотношение $n-t+1 \leqslant\left|\Delta_{1}\right|=1$, откуда следует, что $n=t$, поэтому $\|1\|=\|2\|$. Значит, $\left\|1^{\beta \alpha}\right\|=\left\|2^{\beta \alpha}\right\|$, т.е. мы получаем 3 и 4 , последовательные в $\beta$, в одном блоке, что противоречит I).

III) $n \nmid t$ (по меньшей мере, в случае $d_{1}=\max \left\{d_{1}, \ldots, d_{t}\right\}, d_{2}=\min \left\{d_{1}, \ldots, d_{t}\right\}$ и $\left.d_{3}=\max \left\{d_{3}, \ldots, d_{t}\right\}\right)$. Поэтому $n \neq 2$ и $1^{\alpha} \notin\|1\|$.

ДокАзАтЕльство. Если $n \mid t$, то п. I) и определение перестановки $\beta$ дают $1^{\alpha} \in\|1\|$ (так как $1^{\alpha}=2=1^{\beta^{t}}$ при $d_{1} \neq 1$ ). Поэтому если существует $d_{i}>2$, 
то положим $i=1$ и, применяя $\alpha$ к 1 и $1^{\alpha}=2$, получим последовательные элементы 2 и 3 в $\Delta_{1}$ в одном и том же блоке, что приводит к противоречию с п. I). Если $n \mid t$ и $d_{i} \leqslant 2$ при $i=1, \ldots, t$, то из равенства $\|1\|=\|2\|$ мы получаем противоречие, как и в последней части доказательства п. II). Это доказывает, что $n \nmid t$. Если $n=2$, то условия $n \mid d$ и $n \nmid t$ противоречат условию Гурвица $d \equiv t(\bmod 2)$. Наконец, $1^{\alpha}=2=1^{\beta^{t}} \notin\left\{1^{\beta^{k n}} \mid k \in \mathbb{Z}\right\}=\|1\|$, так как $n \nmid t$.

IV) $\alpha=\left(1 \ldots \delta_{1}\right)\left(\delta_{1}+1 \ldots \delta_{2}\right) \cdots\left(\delta_{t-1}+1 \ldots \delta_{t}\right)$ и $\beta=\left(1 \delta_{1}+1 \ldots \delta_{t-1}+1\right.$ $\left.\Delta_{1} \ldots \Delta_{t}\right)$ индуцируют перестановки $\bar{\alpha}, \bar{\beta}$ системы $\Gamma=\left\{\|1\|,\left\|\delta_{1}+1\right\|, \ldots\right.$, $\left.\left\|\delta_{n-1}+1\right\|\right\}$. По определению перестановки $\beta$ имеем

$$
\bar{\beta}=\left(\|1\|\left\|\delta_{1}+1\right\|\left\|\delta_{2}+1\right\| \ldots\left\|\delta_{n-1}+1\right\|\right) .
$$

Для того чтобы вычислить перестановку $\bar{\alpha}$, достаточно выяснить, в каких блоках находятся элементы подмножеств $\overline{\operatorname{Supp}\left(C_{i}\right)}$ при $i=1, \ldots, t$. Для $C_{1}$ заметим следующее.

V) Каждый элемент блока $\|1\|$ содержится в цикле перестановки $\alpha$ длины большей двух (при условии, что верны те же предположения, что и в пп. II), III)). В частности, $d_{1}>2$.

ДокАЗАТЕЛЬСтво. Если $x \in\|1\| \cap \operatorname{Fix}(\alpha)$, то $1^{\alpha} \in\|1\|$, что противоречит п. III). Если элемент $x \in\|1\|$ содержится в некоторой транспозиции перестановки $\alpha$, то любой элемент из $\|1\|$ тоже содержится в некоторой транспозиции (см. п. I) ) и $d_{i} \leqslant 2$ для любого $i$ (в противном случае $d_{1}>2$, поэтому $1 \in\|1\|$ содержится в $d_{1}$-цикле, не являющемся транспозицией). Поэтому $d_{1}=2$ и $d_{2}=1$. Согласно п. I) и п. II) имеем $\left\|1^{\alpha}\right\|=\left\|\left(\delta_{n}+1\right)^{\alpha}\right\|$, но это невозможно, так как для любого $i$ последовательность $\Delta_{i}$ содержит не более одного элемента, в частности, $\Delta_{1}=\left\{1^{\alpha}\right\}, \Delta_{2}=\varnothing$ и $\Delta_{n+1}=\left\{\left(\delta_{n}+1\right)^{\alpha}\right\}$ (соответственно $\left.\Delta_{n+1}=\varnothing\right)$, поэтому показатель степени перестановки $\beta$, переводящей $1^{\alpha}$ в $\left(\delta_{n}+1\right)^{\alpha}$, меньше $n$ (соответственно равен $n-t$ и в силу п. III) не делится на $n$ ), а потому они представимы различными элементами из $R$ (см. п. I)). Получаем противоречие.

VI) Если $\left\|\delta_{j-1}+1\right\| \cap \operatorname{Supp}\left(C_{1}\right) \neq \varnothing$ и $2 \leqslant j \leqslant t$, то $d_{j}>2$ (при условиях как в пП. II), III)).

ДоказАтельство. Предположим, что $x \in \operatorname{Supp}\left(C_{1}\right) \cap\left\|\delta_{j-1}+1\right\|$ и $d_{j} \leqslant 2$. Так как $d_{1}>2$ в силу п. $\left.\mathrm{V}\right)$, то, применяя последовательно $\alpha^{2}$ к $x$ (соответственно к $\left.\delta_{j-1}+1\right)$, мы получим элементы из $\Delta_{1}$ (соответственно сам элемент $\left.\delta_{j-1}+1\right)$ в одном и том же блоке такие, что показатель степени перестановки $\beta$, переводящей эти элементы друг в друга, не превосходит двух. Получаем противоречие с пп. III), I).

VII) $d_{1}<n$ и $\|1\| \cap \Delta_{1}=\varnothing$ (при условиях как в пп. II), III)). Поэтому разные элементы из $\operatorname{Supp}\left(C_{1}\right)$ принадлежат разным блокам и $\left(\|1\|\|2\| \ldots\left\|d_{1}\right\|\right)$ есть $d_{1}$-цикл перестановки $\bar{\alpha}$.

ДокАЗАТЕЛЬство. Если $d_{1}>n$ (или, по меньшей мере, $\|1\| \cap \Delta_{1} \neq \varnothing$ ), то $n \mid d_{1}$ и $\delta_{t-1}+1 \in\|1\|$, откуда следует, что первый элемент в $\|1\|$, принадлежащий $\Delta_{1}$, есть $n+1$ (так как в противном случае, применяя $\alpha$ к этому элементу и 
к 1 , мы получим в $\Delta_{1}$ элементы из одного блока, которые определяют различные элементы системы $\Gamma$ ). Если $d_{1}=n$, то похожие рассуждения показывают, что $\delta_{t-1}+1 \in\|1\|$. Таким образом,

$$
\beta=(1 \delta_{1}+1 \ldots \delta_{n-1}+1 \ldots \delta_{t-1}+1 \overbrace{2 \ldots n \ldots d_{1}}^{\Delta_{1}} \overbrace{\delta_{1}+2 \ldots \delta_{2}}^{\Delta_{2}} \ldots \Delta_{t})
$$

и в силу п. I) и определения перестановки $\bar{\beta}$ обязательно $\delta_{t-1}+1 \in\|1\|, 2 \in$ $\left\|\delta_{1}+1\right\|$ (поэтому $d_{2}>2$ и $\Delta_{2} \neq \varnothing$ в силу п. $\left.\left.\mathrm{VI}\right)\right), \ldots, n \in\left\|\delta_{n-1}+1\right\|$ и

$$
\bar{\alpha}=\bar{\beta}=\left(\|1\|\left\|\delta_{1}+1\right\|\left\|\delta_{2}+1\right\| \ldots\left\|\delta_{n-1}+1\right\|\right) .
$$

Более того, $d_{1} \in\left\|\delta_{n-1}+1\right\|$ и $\delta_{1}+2 \in\|1\|\left(\right.$ так как $\left.d_{2}>1\right)$, но $\left(\delta_{1}+1\right)^{\alpha}=\delta_{1}+2$, откуда следует, что $\left\|\delta_{1}+1\right\|^{\bar{\alpha}}=\left\|\delta_{1}+2\right\|=\|1\|$ и $n=2$. Получаем противоречие с п. III).

VIII) Цикл из п. VII) может быть представлен как (\|1\| $\left\|\delta_{1}+1\right\|\left\|\delta_{2}+1\right\|$ $\left.\ldots\left\|\delta_{d_{1}-1}+1\right\|\right)$ (при условиях как в пп. II), III)), откуда следует, что $d_{1}=2$, что противоречит п. V).

ДокАЗАТЕЛЬство. Пусть $t=n q+r$ для некоторых $q, r \in \mathbb{Z}^{+}, r<n$ (поэтому $q \geqslant 1$ в силу п. II) и $r \geqslant 1$ в силу п. ІІІ)). Определение перестановки $\beta$ и п. VII) дают $n>r+d_{1}-2$,

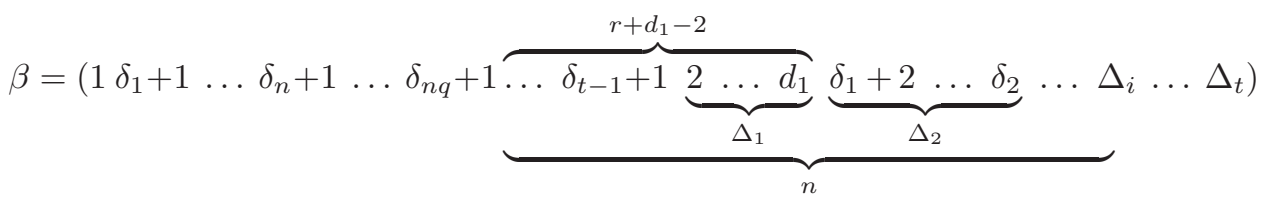

и $\left(\delta_{n q}+1\right)^{\beta^{n}} \in\|1\|$ согласно п. I), где либо $\left(\delta_{n q}+1\right)^{\beta^{n}}=1$ (в случае $d=$ $n q+n$, т.е. при $t>d-n)$, либо $\left(\delta_{n q}+1\right)^{\beta^{n}} \in \Delta_{i}$ для некоторого $i$ из $\{2, \ldots, t\}$ (в случае $d>n q+n$, т.е. при $t<d-n$ ). Предположим, что $d>n q+n$. Отсюда следует, что $\left(\delta_{n q}+1\right)^{\beta^{n}} \in \Delta_{i}$. Согласно п. V) $\Delta_{i}$ содержит более одного элемента и так как $1^{\alpha^{-1}}$ и $\left(\delta_{n q}+1\right)^{\beta^{n} \alpha^{-1}}$ принадлежат одному блоку, то обязательно $\left(\delta_{n q}+1\right)^{\beta^{n}}=\delta_{i-1}+2$ (первый элемент в $\left.\Delta_{i}\right)$. Поэтому $1^{\alpha \beta^{-1}}$ и $\left(\delta_{n q}+1\right)^{\beta^{n} \alpha \beta^{-1}}$ принадлежат $\|1\|^{\bar{\alpha} \bar{\beta}^{-1}}$, но $\left(\delta_{n q}+1\right)^{\beta^{n} \alpha \beta^{-1}}=\delta_{i-1}+2$. Значит, $\|1\|^{\bar{\alpha} \bar{\beta}^{-1}}=\|1\|, 2 \in\left\|\delta_{1}+1\right\|$ и в силу определений перестановок $\alpha$ и $\beta$ цикл $\left(\|1\|\left\|\delta_{1}+1\right\|\left\|\delta_{2}+1\right\| \ldots\left\|\delta_{d_{1}-1}+1\right\|\right)$ является циклом перестановки $\bar{\alpha}$. Поэтому $2 \in\left\|\delta_{1}+1\right\|$ (откуда $d_{2}>2$ в силу п. VI) $), d_{1} \in\left\|\delta_{d_{1}-1}+1\right\|$ и $\delta_{1}+2 \in\left\|\delta_{d_{1}}+1\right\|$. Но $\delta_{1}+2=\left(\delta_{1}+1\right)^{\alpha}$, откуда получаем, что $\left\|\delta_{2}+1\right\|=\left\|\delta_{1}+1\right\|^{\bar{\alpha}}=\left\|\delta_{d_{1}}+1\right\|$. Поэтому $n \mid\left(d_{1}-2\right)$ в силу п. I) и, следовательно, $d_{1}=2$ в силу п. VII). Предположим, что $d=n q+n$. Отсюда следует, что $t>d-n$ и $\left(\delta_{n q}+1\right)^{\beta^{n}}=1$. Так как $\delta_{n q}+1 \in\|1\|$, то из п. V) следует, что $\Delta_{n q+1} \neq \varnothing$ и $\left(\delta_{n q}+1\right)^{\alpha} \in \Delta_{n q+1}$. Поэтому $1^{\alpha}$ и $\left(\delta_{n q}+1\right)^{\alpha}$ являются различными элементами последовательностей $\Delta_{1}$ и $\Delta_{n q+1}$. Поэтому показатель степени перестановки $\beta$, переводящей эти элементы друг в друга, меньше чем $\left|\Delta_{1}\right|+\cdots+\left|\Delta_{t}\right|=d-t<n$. Из п. I) следует, что эти два элемента принадлежат разным блокам. Получаем противоречие. Утверждение п. VIII) доказано. 
Таким образом, любой блок группы $H$ тривиален и $H$ примитивна. Наконец, так как $H<G_{1}=\langle\lambda, \beta\rangle$, то группа $G_{1}$ тоже примитивна и случай поверхности $T_{1}$ разобран. Для поверхности $P_{2}$ мы используем идею, описанную в наброске доказательства в начале этого параграфа, чтобы определить $\omega, \theta$ и $G_{2}=\langle\omega, \theta\rangle$. Таким образом, подгруппа $G_{2}$ примитивна, так как она содержит $H$. Теорема 3.2 доказана.

Второй автор выражает благодарность Е. Кудрявцевой и С. Богатому из МГУ им. М. В. Ломоносова за многочисленные полезные и плодотворные обсуждения описанных выше задач во время своего визита на кафедру Дифференциальной геометрии и приложений механико-математического факультета МГУ в мае 2002г. Он также благодарит коллектив этой кафедры за гостеприимство во время своего визита. Авторы также выражают благодарность рецензенту за многочисленные комментарии и предложения, которые значительно улучшили изложение и качество этой работы, в частности, за финальную часть доказательства п. VIII) доказательства теоремы 3.2, восполняющую пробел в исходном доказательстве.

\section{Список литературы}

[1] K. Borsuk, R. Molski, "On a class of continuous mappings", Fund. Math., 45 (1957), $84-98$.

[2] K. Sieklucki, "On superpositions of simple mappings", Fund. Math., 48 (1960), $217-228$.

[3] J. D. Baildon, "Open simple maps and periodic homeomorphisms", Proc. Amer. Math. Soc., 39 (1973), 433-436.

[4] J. Krzempek, "Covering maps that are not compositions of covering maps of lesser order", Proc. Amer. Math. Soc., 130:6 (2002), 1867-1873.

[5] С. И. Богатая, С. А. Богатый, Х. Цишанг, "О композициях открытых отображений", Матем. сб., 193:3 (2002), 3-20; англ. пер.: S. I. Bogataya, S. A. Bogatyi, H. Zieschang, "On compositions of open mappings", Sb. Math., 193:3 (2002), 311-327.

[6] G. Th. Whyburn, Analytic topology, Amer. Math. Soc., Providence, RI, 1963.

[7] S. Bogatyi, D. L. Gonçalves, H. Zieschang, "The minimal number of roots of surface mappings and quadratic equations in free groups", Math. Z., 236:3 (2001), 419-452.

[8] D. H. Husemoller, "Ramified coverings of Riemann surfaces", Duke Math. J., 29 (1962), 167-174.

[9] C. L. Ezell, "Branch point structure of covering maps onto nonorientable surfaces", Trans. Amer. Math. Soc., 243 (1978), 123-133.

[10] S. Bogatyi, D. L. Gonçalves, E. Kudryavtseva, H. Zieschang, "Realization of primitive branched coverings over closed surfaces following the Hurwitz approach", Cent. Eur. J. Math., 1:2 (2003), 184-197.

[11] S. A. Bogatyi, D. L. Gonçalves, E. A. Kudryavtseva, H. Zieschang, "Realization of primitive branched coverings over closed surfaces", Advances in topological quantum field theory (Kananaskis Village, Canada, 2001), NATO Sci. Ser. II Math. Phys. Chem., 179, Kluwer Acad. Publ., Dordrecht, 2004, 297-316.

[12] A. L. Edmonds, R.S. Kulkarni, R.E. Stong, "Realizability of branched coverings of surfaces", Trans. Amer. Math. Soc., 282:2 (1984), 773-790.

[13] D. L. Gonçalves, E. Kudryavtseva, H. Zieschang, "Roots of mappings on nonorientable surfaces and equations in free groups", Manuscripta Math., 107:3 (2002), 311-341. 
[14] P. Müller, "Primitive monodromy groups of polynomials", Recent developments in the inverse Galois problem (Seattle, WA, USA, 1993), Contemp. Math., 186, Amer. Math. Soc., Providence, RI, 1995, 385-401.

[15] R. M. Guralnick, M. G. Neubauer, "Monodromy groups of branched coverings: The generic case", Recent developments in the inverse Galois problem (Seattle, WA, USA, 1993), Contemp. Math., 186, Amer. Math. Soc., Providence, RI, 1995, 325-352.

[16] S. K. Lando, A. K. Zvonkin, "Graphs on surfaces and their applications", Low-dimensional topology. v. II, Encyclopaedia Math. Sci., 141, Springer-Verlag, Berlin, 2004.

[17] J.D. Dixon, B. Mortimer, Permutation groups, Grad. Texts in Math., 163, Springer-Verlag, New York, 1996.

[18] A. Hurwitz, "Ueber Riemann'sche Flächen mit gegebenen Verzweigungspunkten", Math. Ann., 39:1 (1891), 1-60.

[19] J. F. Ritt, "Prime and composite polynomials", Trans. Amer. Math. Soc., 23:1 (1922), $51-66$.

[20] N. Jacobson, Basic algebra. I, Freeman, New York, 1985.

Н. А. В. Бедоя (N. A. V. Bedoya)

Federal University of São Carlos, Brasil

E-mail: nbedoya@dm.ufscar.br

Д. Л. Гонсалвес (D. L. Gonçalves)

Federal University of São Carlos, Brasil

E-mail: dlgoncal@ime.usp.br
Поступила в редакцию 27.04 .2009 и 17.04 .2010 